\title{
Receptor binding and priming of the spike protein of SARS-CoV-2 for membrane fusion
}

https://doi.org/10.1038/s41586-020-2772-0

Received: 1 July 2020

Accepted: 11 September 2020

Published online: 17 September 2020

Check for updates

\author{
Donald J. Benton ${ }^{1,6 凶}$, Antoni G. Wrobel ${ }^{1,6 凶}$, Pengqi Xu ${ }^{2,3}$, Chloë Roustan ${ }^{4}$, Stephen R. Martin', \\ Peter B. Rosenthal ${ }^{5}$, John J. Skehel ${ }^{1}$ \& Steven J. Gamblin ${ }^{1 凶}$
}

Infection with severe acute respiratory syndrome coronavirus 2 (SARS-CoV-2) is initiated by virus binding to the ACE2 cell-surface receptors ${ }^{1-4}$, followed by fusion of the virus and cell membranes to release the virus genome into the cell. Both receptor binding and membrane fusion activities are mediated by the virus spike glycoprotein $^{5-7}$. As with other class-I membrane-fusion proteins, the spike protein is post-translationally cleaved, in this case by furin, into the $\mathrm{S} 1$ and $\mathrm{S} 2$ components that remain associated after cleavage ${ }^{8-10}$. Fusion activation after receptor binding is proposed to involve the exposure of a second proteolytic site (S2'), cleavage of which is required for the release of the fusion peptide $\mathrm{e}^{11,12}$. Here we analyse the binding of ACE2 to the furin-cleaved form of the SARS-CoV-2 spike protein using cryo-electron microscopy. We classify ten different molecular species, including the unbound, closed spike trimer, the fully open ACE2-bound trimer and dissociated monomeric S1 bound to ACE2. The ten structures describe ACE2-binding events that destabilize the spike trimer, progressively opening up, and out, the individual $S 1$ components. The opening process reduces $\mathrm{S} 1$ contacts and unshields the trimeric $\mathrm{S} 2$ core, priming the protein for fusion activation and dissociation of ACE2-bound S1 monomers. The structures also reveal refolding of an $\mathrm{S} 1$ subdomain after ACE2 binding that disrupts interactions with S2, which involves Asp $614^{13-15}$ and leads to the destabilization of the structure of S2 proximal to the secondary (S2') cleavage site.
Recognition of the ACE2 receptor by the membrane spike glycoprotein of SARS-CoV-2 is a major determinant of virus infectivity, pathogenesis and host range. Previous structural studies on the spike glycoproteins of coronaviruses ${ }^{6,16-22}$ have shown that the spike trimer consists of a central helical stalk-comprising three interacting S2 components-that is covered at the top by S1. Each S1 component consists of two large domains, the N-terminal domain (NTD) and receptor-binding domain (RBD), each associated with a smaller intermediate subdomain. In virus membranes, spike glycoproteins exist in a closed form, in which the RBDs cap the top of the S2 core and are inaccessible to ACE2, and in an open form, in which one S1 component has opened to expose the RBD for ACE2 binding ${ }^{6,16,18,23}$. Recent structural studies ${ }^{7,24,25}$ on the isolated RBD of the SARS-CoV-2 spike protein in complex with ACE2 have provided a molecular description of the receptor-binding interface. Although some comparisons can be inferred from the previous cryo-electron microscopy studies on the spike protein of SARS-CoV ${ }^{12,18,19,23}$, structures of intact trimeric SARS-CoV-2 spike with bound ACE2 are needed to determine the effects of binding on the overall spike conformation.

To examine this interaction between the SARS-CoV-2 spike protein and its receptor, we mixed the ectodomains of furin-cleaved spike with the ectodomains of ACE2 and incubated them for around $60 \mathrm{~s}$ before plunge-freezing the mixture in liquid ethane for examination by cryo-electron microscopy. In the images that we obtained, we could resolve ten distinct species of spike and spike-ACE2 complexes (Fig. 1 and Extended Data Fig. 1), ranging from tightly closed, unbound trimers to open trimers that formed complexes with three ACE2 molecules and dissociated monomeric S1-ACE2 complexes. Of the spike trimers analysed, two thirds were bound to ACE2 (Extended Data Fig. 1). Of the unbound species, we observe good-quality particles in the closed unbound conformation, equally compact to those reported in our previous study ${ }^{26}$ and slightly more so than those described in previous reports $^{6,16}$. There are also considerable numbers ( $16 \%$ of all trimers) of unbound particles with one erect RBD, as well as some (4\%) in an intermediate conformation, a less-compact closed form, with a single disordered RBD, which have also been reported in a previous study of the furin-cleaved spike protein ${ }^{26}$.

Of the spike trimers bound to the receptor, half accommodate one ACE2 receptor. As previously reported for the SARS-CoV spike protein $^{12,23}$, the ACE2-bound RBD occupies a range of tilts with respect to the long axis of the trimer (Extended Data Fig. 2a). Of the two RBDs per trimer that are not engaged with the receptor, either both are closed or one of the RBDs remains closed and one (either clockwise or anticlockwise to the bound S1 (Extended Data Fig.1)) is in the open conformation. We were also able to identify, reconstruct and refine trimers to which two or three ACE2 receptors were bound, in successively more open structures (Fig. 1 and Extended Data Fig. 1).

${ }^{1}$ Strutural Biology of Disease Processes Laboratory, Francis Crick Institute, London, UK. ${ }^{2}$ Precision Medicine Center, The Seventh Affiliated Hospital, Sun Yat-sen University, Shenzhen, China. ${ }^{3}$ Francis Crick Institute, London, UK. ${ }^{4}$ Structural Biology Science Technology Platform, Francis Crick Institute, London, UK. ${ }^{5}$ Structural Biology of Cells and Viruses Laboratory, Francis Crick Institute, London, UK. ${ }^{6}$ These authors contributed equally: Donald J. Benton, Antoni G. Wrobel. ${ }^{\bowtie}$ e-mail: donald.benton@crick.ac.uk; antoni.wrobel@crick.ac.uk; steve.gamblin@crick.ac.uk 


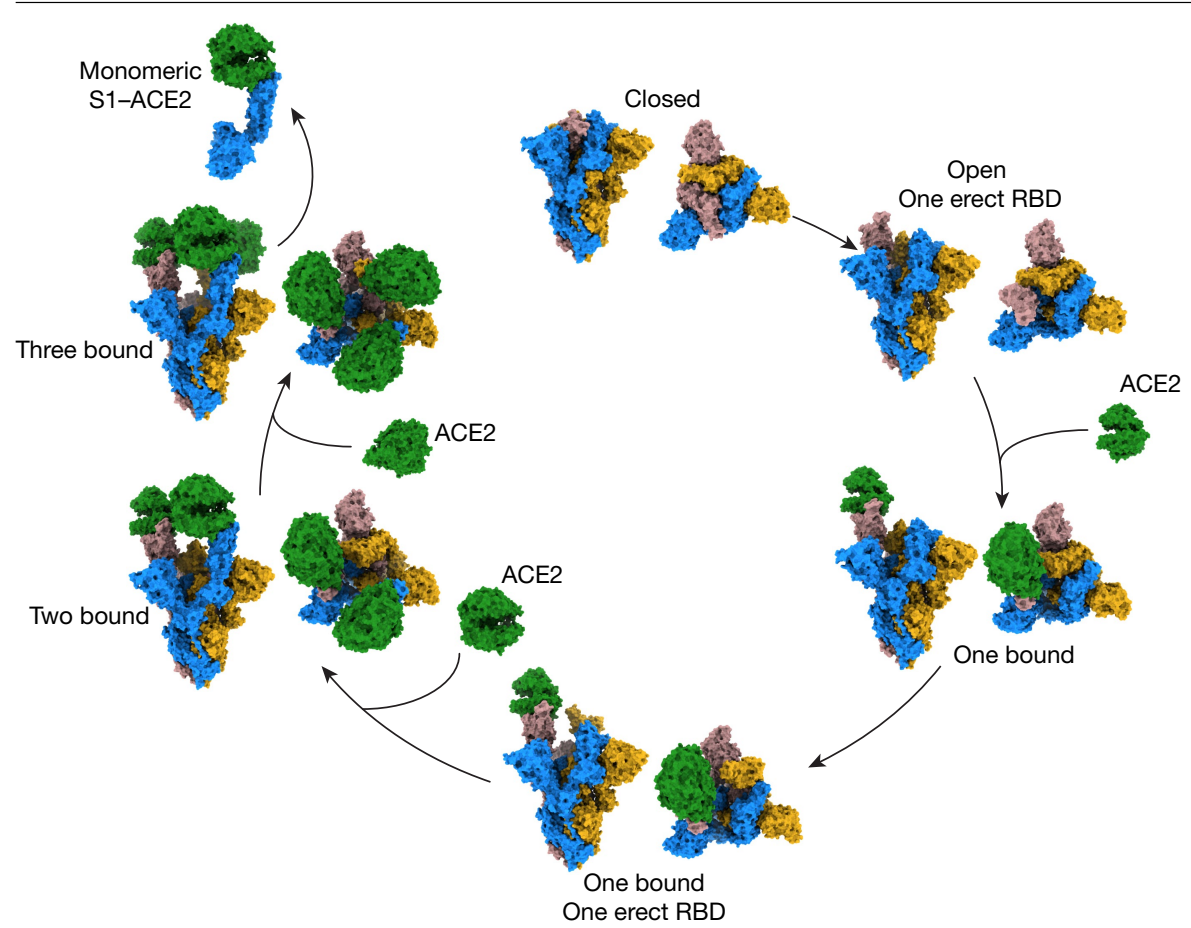

Fig. 1 | Sequential steps in ACE2 binding of the SARS-CoV-2 spike protein. Surface representation of the spike, with monomers coloured in blue, rosy brown and gold, and ACE2 coloured in green. Each step shows two views of the spike complexes: a trimer axis vertical view (left) and an orthogonal top-down view along the axis (right). Clockwise from the top, we show structures for closed, open but unbound RBD, followed by sequential ACE2-binding events until reaching the fully open, three-ACE2-bound spike protein state. From this final trimeric species, we show dissociation into monomeric S1-ACE2, which may also occur for the one- or two-ACE2-bound species.

Comparison of the trimers with one erect RBD that is either bound or unbound by an ACE2 receptor revealed two things. First, ACE2 binding alters the position of the open RBD by a rigid-body rotation of the domain that moves its centre of mass on average a further approximately $5.5 \AA$ away from the trimer axis, the NTD-associated and RBD-associated subdomains of the same monomer shift around $1.9 \AA$ and about 2.3 $\AA$, respectively (Extended Data Fig. 2c), and at the same time the NTDs of all three S1 components move by around 1.5-3.0
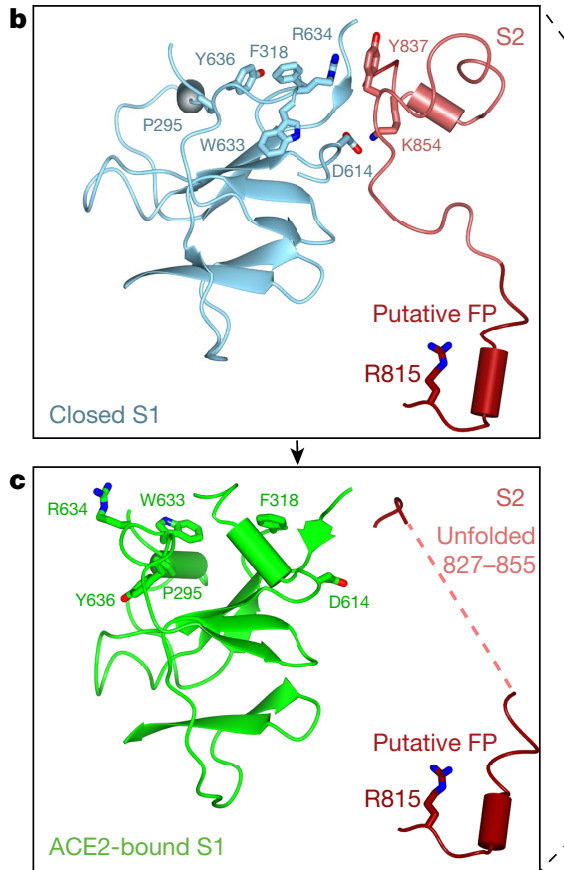

Fig. 2 | Structural rearrangements between the closed and the ACE2-bound states of the spike protein. a, Surface representation of a monomer of S2 in the one-ACE2-bound, two-RBD-closed state coloured in light pink with the S1 subunit of the adjacent monomer in ribbon representation; the S1 of the one-ACE2-bound, two-RBD-closed state is shown in green and the three-RBDclosed state $\left(\mathrm{PDB} 6 \mathrm{ZGE}^{26}\right)$ is shown in blue. The atoms on the surface of S2 that contact the $\mathrm{S} 1$ intermediate domains are coloured in red. The arrows indicate the direction of movements of the intermediate domains, and of the RBD, between the closed and ACE2-bound conformations of the spike. b, Ribbon representations of the NTD-associated intermediate domain in blue and the

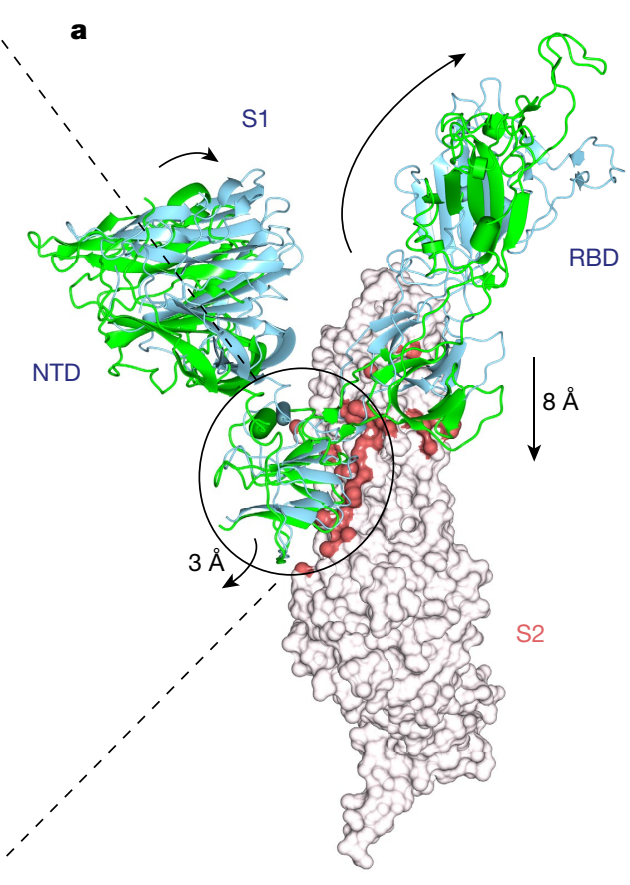

moiety of the S2 chain that it interacts with (in red) in the closed conformation of the spike. Essential residues that participate in the interaction are labelled; of particular note is the salt bridge between Asp614 (S1, chain A) and Lys854 $(\mathrm{S} 2$, chain $\mathrm{B}) . \mathbf{c}$, Ribbon representation of the same intermediate domain as in $\mathbf{b}$, but in the conformation observed in the ACE2-bound structure of the spike (in green), in which the movement and refolding of the domain leads to a loss of interaction with $\mathrm{S} 2$, which becomes disordered. The putative fusion peptide (FP) and the S2' site of the second protease cleavage at R815 adjacent to the region that undergoes unfolding are shown in dark red. 

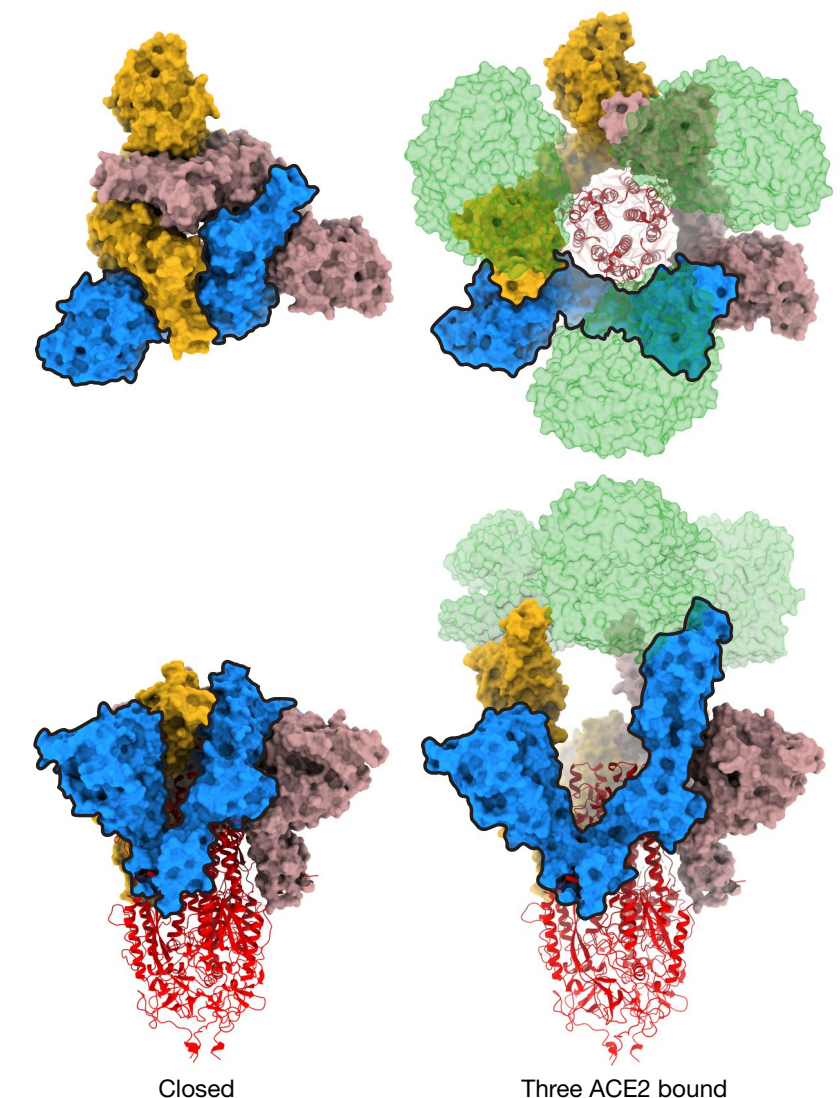

Three ACE2 bound

Fig. 3 | Structural basis of S2 unsheathing by ACE2 binding. The spike protein is shown as a space-filling representation for $\mathrm{S1}$, with each monomer coloured blue, rosy brown and gold, and as a ribbon representation for S2 coloured in red for all three monomers. Left, top-down and side-on views of the trimer in the closed conformation. Right, the same views for the fully open three-ACE2-bound species.

(Extended Data Fig. 2d). Similar changes in the domain orientation are observed in the recent structure of the SARS-CoV-2 spike complex with

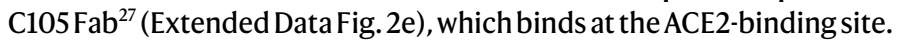
However, the molecular basis of both of these sets of changes remains unclear. Binding of more than one ACE2 molecule does not induce any substantial further changes in the average positioning of the RBD (Extended Data Fig. 2e). Second, our data suggest that ACE2 binding favours the open conformation of the RBD. The relatively high-affinity interaction of RBD with ACE2 generates an RBD-ACE2 structure that cannot be accommodated in a closed trimer-the bound state does not have access to the closed conformation. In addition, the fact that ACE2 binding induces a more-open conformation of the spike RBD suggests that some of the binding energy is used to drive the new conformation of $\mathrm{S} 1$, which is then further excluded from a closed state.

The successive steps, from closed unbound trimer to the fully open, three-ACE2-bound trimer, are associated with a substantial reduction in the contact area that each S1 makes with both its neighbouring S1 monomers and with the S2 trimeric core (Extended Data Table 1). For the fully, three-ACE2-bound species, each S1 makes $1,400 \AA^{2}$ less contact with both its $S 1$ trimer neighbours and $1,300 \AA^{2}$ less contact with the $S 2$ core than in the fully closed trimer conformation; all of these rearrangements are driven by the energetics of the three ACE2-binding events. The movements of the RBD and NTD domains of S1 that are associated with the opening of the structure and stabilization of the new arrangement by ACE2 binding, as described above, leave a trimeric ring of S1 molecules that are attached to the S2 core only through contacts with its two small intermediate subdomains (Fig. 2a). Comparing the ACE2-bound, open form (the open-unbound structure is
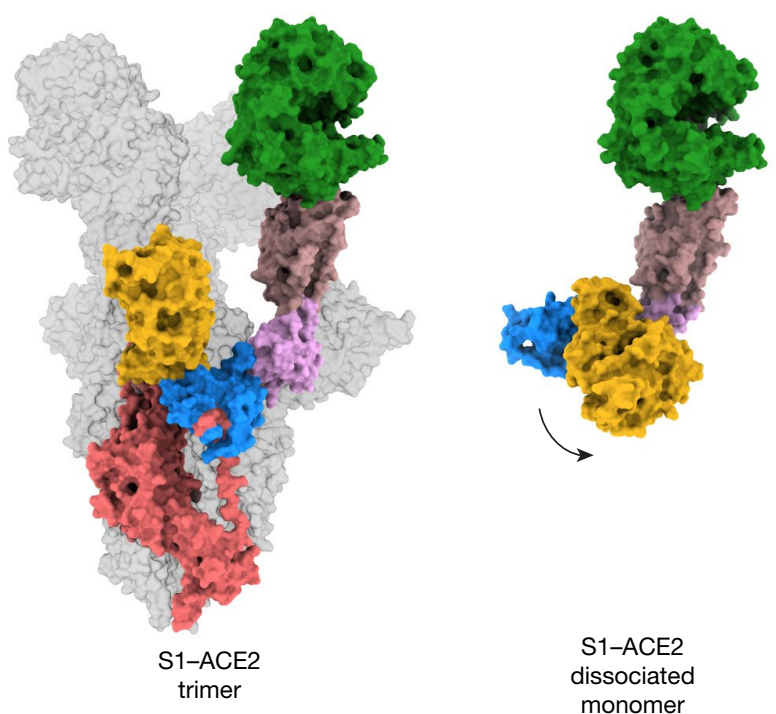

Fig. 4 | ACE2-bound S1 subunit as a part of the spike trimer and as an isolated monomer. Space-filling representations of the spike protein with one monomer coloured polychromatically. NTD, yellow; NTD-associated subdomain, blue; RBD-associated subdomain, pink; RBD, rosy brown; S2, red; ACE2, green. The remainder of the trimer on the left is coloured grey. The structure on the right is aligned on the RBD:ACE2 moiety of the trimer complex on the left. The arrow indicates the direction of movement of the NTD and NTD-associated subdomain on the transition from the trimer (left) to the monomer species (right).

similar but of poorer local resolution) with the fully closed trimer, the RBD-associated intermediate subdomain moves about $8 \AA$, whereas the NTD-associated intermediate subdomain moves by $3 \AA$ (Fig. 2a). The latter also undergoes a partial restructuring with possibly important implications for the mechanism of fusion activation of spike. In the closed form, one edge of the NTD-associated intermediate subdomain interacts with a short helix and a loop from S2 of the neighbouring monomer (Fig. 2b). Notably, two components of this interaction comprise a series of side-chain $\pi$-stacking interactions in the closed structure $^{26}$ : Tyr636, Phe318 and Arg634 of S1 with Tyr837 of S2; and a salt bridge formed by Asp614 of S1 with Lys854 of S2. By contrast, in the ACE2-bound form, Tyr636, Phe318 and Trp633 refold to the side of the domain further away from the symmetry axis (as viewed in Fig. 2c), leaving a channel to accommodate a new segment of $\alpha$-helix that forms downstream of Asp614 from polypeptide chain that was previously disordered. As a consequence, the interactions between S1 and S2 described above for the closed form are lost in the ACE2-bound form and the segment comprising residues 827-855 of S2 becomes disordered (Fig. 2c). This part of S2 is immediately C-terminal to the putative fusion peptide of $\mathrm{S} 2^{11}$, the $\mathrm{N}$ terminus of which is defined by Arg815 at the S2' cleavage site ${ }^{9,11}$. The opening of the ACE2-stabilized S1 therefore leads to the destabilization of the S2 structure just after the putative fusion peptide, potentially activating it for exposure in the next stages of membrane fusion. Notably, Asp614, which forms salt bridges to Lys 854 of S2 in the closed form, is frequently substituted ${ }^{13-15}$ by a glycine residue and it has been suggested that this substitution reduces shedding of $\mathrm{S} 1$ (and increases the number of spike proteins on the virus surface $)^{13}$. We also propose that this substitution would remove a key salt bridge, and that the unique stereochemistry available to glycine may facilitate the formation of the new segment of $\alpha$-helix, which is also incompatible with the $\mathrm{S} 2$ interaction. Furthermore, it could lead to reduced stability of the closed form of the spike protein, which in turn would increase the likelihood of the RBDs adopting the open conformation and hence the ability of the spike protein to bind to ACE2.

The opening up, and out, from the trimer axis of the S1 domains after ACE2 binding gives rise to an unshielding of the top surface of 
the helix-loop-helix (approximately residues 980-990 within the HR1 region ${ }^{20,22,28,29}$ ) at the top of the S2 domain (Fig. 3). In the closed form, these helices and their connecting turns are tightly shielded by the RBDs; each S2 monomer is predominantly covered by its anticlockwise-related S1 trimer neighbour. In the fully open state, the S1 domains move in such a way as to generate a cavity with a diameter of $50 \AA$ around the trimer axis that is about $65 \AA$ deep. At the bottom of this cavity is the now solvent-exposed, central portion of HR1. For membrane fusion to occur-in comparison with other class-I fusion proteins and as described in coronavirus post-fusion structures ${ }^{22,28,29}$ the $\mathbf{S} 2$ component is likely to undergo a major helical rearrangement, in which the long trimer interface helix (spanning residues 990-1035) grows and extends, by incorporating the refolded turn and helix from the $\mathrm{N}$-terminal portion of HR1, and projects the fusion peptide towards the host cell membrane. In this process, opening up of all three S1 monomers and their subsequent dissociation would enable the concerted helical refolding, as the cooperative displacement of the capping portions of the protein will probably be required for the extension of the helical coil, as has recently been observed for the haemagglutinin protein of influenza ${ }^{30}$. The stoichiometry of S1 subunit-ACE2 interactions required for effective cell-surface contact or for priming is not addressed by our experiments. However, as the affinity of individual monomers for ACE2 appears to be sufficient for cellular association, it may be that more than one subunit is required to be in the open form for efficient priming of these rearrangements in S2 that occur in the process of membrane fusion. It seems reasonable to propose that the likelihood of triggering the fusion conformation increases with the number of ACE2 receptors bound.

In addition to the range of species of trimeric spike described above, the largest single population of particles that we were able to identify and reconstruct represent ACE2 bound to a S1 monomer (Fig. 4). The interaction between $\mathrm{ACE} 2$ and the RBD, and the interaction of the latter with its associated intermediate subdomain, are very similar between the monomeric and trimer versions and with previously determined crystal and electron microscopy structures of ACE2 and RBD $7,24,25$. However, there are increasingly large rearrangements between the two intermediate subdomains and then with the NTD. By applying non-uniform refinement, the highest resolution was achieved for the reconstruction of the ACE2-RBD interaction (Extended Data Fig. 4), in part because of the tight interaction but also probably because of the dominant influence of this part of the structure on the alignment process. Nevertheless, it is clear that there are both increasingly large changes in the interfaces between domains on moving towards the NTD and a range of subpopulations of related but variable conformations. The high proportion of ACE2-S1 monomers, and the limited contact areas between the trimeric S1 ring interactions with S2, suggest that the fully open ACE2-bound spike complex is probably metastable.

Taken together, our structural data enable mechanistic suggestions for the early stages of SARS-CoV-2 infection of cells. The SARS-CoV-2 spike protein is produced in a compact closed form in which the helices in the S2 membrane fusion component are capped by the RBD of neighbouring monomers. After cleavage by furin between the S1 and S2 domains, the proportion of the spike trimers that is able to accommodate RBD in an open, ACE2-binding conformation increase ${ }^{26}$. Binding of the ACE2 receptor to an open RBD leads to a more-open trimer conformation. The geometry of ACE2 binding is incompatible with the RBD adopting a closed conformation and leads to our observation of several two-open-RBD conformations as well as the three-RBD-bound conformation. Successive RBD opening and ACE2 binding lead to a fully open and ACE2-bound form in which the trimeric S1 ring remains bound to the core S2 trimer by limited contacts through the intermediate subdomains of S1. This arrangement leaves the top of the S2 helices fully exposed. In the process, the interaction of the closed form of S1 with a segment of the $\mathrm{S} 2$ chain that precedes the putative fusion peptide region, in the open form, is lost. We suggest that in this form the $\mathrm{S}$ trimer is primed for the helical rearrangements of S2 that are required for fusion of the viral and host cell membranes ${ }^{28}$.

\section{Online content}

Any methods, additional references, Nature Research reporting summaries, source data, extended data, supplementary information, acknowledgements, peer review information; details of author contributions and competing interests; and statements of data and code availability are available at https://doi.org/10.1038/s41586-020-2772-0.

1. Zhou, P. et al. A pneumonia outbreak associated with a new coronavirus of probable bat origin. Nature 579, 270-273 (2020)

2. Wan, Y., Shang, J., Graham, R., Baric, R. S. \& Li, F. Receptor recognition by the novel coronavirus from Wuhan: an analysis based on decade-long structural studies of SARS coronavirus. J. Virol. 94, e00127-20 (2020).

3. Li, F., Li, W., Farzan, M. \& Harrison, S. C. Structure of SARS coronavirus spike receptor-binding domain complexed with receptor. Science 309, 1864-1868 (2005).

4. Li, W. et al. Angiotensin-converting enzyme 2 is a functional receptor for the SARS coronavirus. Nature 426, 450-454 (2003).

5. Li, F. Structure, function, and evolution of coronavirus spike proteins. Annu. Rev. Virol. 3 , 237-261 (2016).

6. Walls, A. C. et al. Structure, function, and antigenicity of the SARS-CoV-2 spike glycoprotein. Cell 181, 281-292 (2020).

7. Shang, J. et al. Structural basis of receptor recognition by SARS-CoV-2. Nature $\mathbf{5 8 1}$, 221-224 (2020).

8. Belouzard, S., Chu, V. C. \& Whittaker, G. R. Activation of the SARS coronavirus spike protein via sequential proteolytic cleavage at two distinct sites. Proc. Natl Acad. Sci. USA 106, 5871-5876 (2009).

9. Millet, J. K. \& Whittaker, G. R. Host cell entry of Middle East respiratory syndrome coronavirus after two-step, furin-mediated activation of the spike protein. Proc. Natl Acad. Sci. USA 111, 15214-15219 (2014).

10. Hoffmann, M. et al. SARS-CoV-2 cell entry depends on ACE2 and TMPRSS2 and is blocked by a clinically proven protease inhibitor. Cell 181, 271-280 (2020)

11. Lai, A. L., Millet, J. K., Daniel, S., Freed, J. H. \& Whittaker, G. R. The SARS-CoV fusion peptide forms an extended bipartite fusion platform that perturbs membrane order in a calcium-dependent manner. J. Mol. Biol. 429, 3875-3892 (2017).

12. Song, W., Gui, M., Wang, X. \& Xiang, Y. Cryo-EM structure of the SARS coronavirus spike glycoprotein in complex with its host cell receptor ACE2. PLoS Pathog. 14, e1007236 (2018).

13. Zhang, L. et al. The D614G mutation in the SARS-CoV-2 spike protein reduces S1 shedding and increases infectivity. Preprint at https://doi.org/10.1101/2020.06.12.148726 (2020).

14. Hu, J. et al. D614G mutation of SARS-CoV-2 spike protein enhances viral infectivity. Preprint at https://doi.org/10.1101/2020.06.20.161323 (2020).

15. Korber, B. et al. Tracking changes in SARS-CoV-2 spike: evidence that D614G increases infectivity of the COVID-19 virus. Cell 182, 812-827 (2020).

16. Wrapp, D. et al. Cryo-EM structure of the 2019-nCoV spike in the prefusion conformation. Science 367, 1260-1263 (2020).

17. Tortorici, M. A. et al. Structural basis for human coronavirus attachment to sialic acid receptors. Nat. Struct. Mol. Biol. 26, 481-489 (2019).

18. Yuan, Y. et al. Cryo-EM structures of MERS-CoV and SARS-CoV spike glycoproteins reveal the dynamic receptor binding domains. Nat. Commun. 8, 15092 (2017).

19. Kirchdoerfer, R. N. et al. Stabilized coronavirus spikes are resistant to conformational changes induced by receptor recognition or proteolysis. Sci. Rep. 8, 15701 (2018).

20. Pallesen, J. et al. Immunogenicity and structures of a rationally designed prefusion MERS-CoV spike antigen. Proc. Natl Acad. Sci. USA 114, E7348-E7357 (2017).

21. Walls, A. C. et al. Cryo-electron microscopy structure of a coronavirus spike glycoprotein trimer. Nature 531, 114-117 (2016).

22. Cai, Y. et al. Distinct conformational states of SARS-CoV-2 spike protein. Science $\mathbf{3 6 9}$ 1586-1592 (2020).

23. Gui, M. et al. Cryo-electron microscopy structures of the SARS-CoV spike glycoprotein reveal a prerequisite conformational state for receptor binding. Cell Res. 27, 119-129 (2017).

24. Lan, J. et al. Structure of the SARS-CoV-2 spike receptor-binding domain bound to the ACE2 receptor. Nature 581, 215-220 (2020).

25. Yan, R. et al. Structural basis for the recognition of SARS-CoV-2 by full-length human ACE2. Science 367, 1444-1448 (2020).

26. Wrobel, A. G. et al. SARS-CoV-2 and bat RaTG13 spike glycoprotein structures inform on virus evolution and furin-cleavage effects. Nat. Struct. Mol. Biol. 27, 763-767 (2020).

27. Barnes, C. O. et al. Structures of human antibodies bound to SARS-CoV-2 spike reveal common epitopes and recurrent features of antibodies. Cell 182, 828-842 (2020).

28. Walls, A. C. et al. Tectonic conformational changes of a coronavirus spike glycoprotein promote membrane fusion. Proc. Natl Acad. Sci. USA 114, 11157-11162 (2017).

29. Fan, X., Cao, D., Kong, L. \& Zhang, X. Cryo-EM analysis of the post-fusion structure of the SARS-CoV spike glycoprotein. Nat. Commun. 11, 3618 (2020).

30. Benton, D. J., Gamblin, S. J., Rosenthal, P. B. \& Skehel, J. J. Structural transitions in influenza haemagglutinin at membrane fusion pH. Nature 583, 150-153 (2020).

Publisher's note Springer Nature remains neutral with regard to jurisdictional claims in published maps and institutional affiliations.

(c) The Author(s), under exclusive licence to Springer Nature Limited 2020 


\section{Methods}

\section{Constructs design, protein expression and purification}

The ectodomains of ACE2 (19-615) and stabilized, '2P' mutant (K986P and V987P) of SARS-CoV-2 spike (residues 1-1208) with intact furin-cleavage site were prepared as described in a recent study ${ }^{26}$. In brief, the proteins were expressed in Expi293F cells (Gibco), collected twice after 3-4 and 6-7 days, and purified with affinity chromatography (spike using CoNTA resin from TAKARA, ACE2 with Streptactin XT resin from IBA Lifesciences), followed by gel filtration into a buffer containing $20 \mathrm{mM}$ Tris pH 8.0 and $150 \mathrm{mM} \mathrm{NaCl}$. As previously described ${ }^{26}$, the purified spike was then incubated for $5 \mathrm{~h}$ with exogenous furin (New England Biolabs), after which the reaction was stopped by addition of EDTA.

\section{Electron microscopy sample preparation and data collection}

R2/2 200-mesh Quantifoil grids were glow-discharged for $30 \mathrm{~s}$ at $25 \mathrm{~mA}$ to prepare them for freezing. The furin-treated SARS-CoV-2 spike was mixed with octyl glucoside as previously described ${ }^{26}$ and, 45-60 s before ultimately plunge-freezing the grid, with concentrated ACE2 at a 1:2 final molar ratio of trimeric spike:ACE2, aiming to obtain a final concentration of spike of $0.5 \mathrm{mg} \mathrm{ml}^{-1}$ and octyl glucoside of $0.1 \%$. Then, $4 \mu \mathrm{l}$ of the obtained reaction mixture was applied on a grid pre-equilibrated at $4{ }^{\circ} \mathrm{C}$ in $100 \%$ humidity, blotted with filter paper for 4-4.5 s using Vitrobot Mark III, and plunge-frozen in liquid ethane.

Data were collected using EPU software on a Titan Krios microscope operating at $300 \mathrm{kV}$. Micrographs were collected using a Gatan K2 detector mounted on a Gatan GIF Quantum energy filter operating in zero-loss mode with a slit width of $20 \mathrm{eV}$. Exposures were $8 \mathrm{~s}$, fractionated into 32 frames with an accumulated dose of $54.4 e^{-} \AA^{-2}$, with a calibrated pixel size of 1.08 Å. Images were collected at a range of defocibetween 1.5 and $3.0 \mu \mathrm{m}$.

\section{Electron microscopy data processing}

Movies were aligned using MotionCor $2^{31}$ implemented in $\mathrm{RELION}^{32}$, followed by contrast transfer function (CTF) estimation using Ctffind $4^{33}$. Particles were picked using crYOLO ${ }^{34}$ using a manually trained model. Particles were subjected to multiple rounds of two-dimensional classification using cryoSPARC ${ }^{35}$. Classes that displayed a clear secondary structure were retained and split into subsets, which either resembled spike trimers or S1 monomers bound to ACE2. Initial models were made using the ab initio reconstruction in cryoSPARC. Different species containing trimeric spike proteins were separated by extensive three-dimensional classification in RELION as shown in Extended Data Fig. 3. Before the final refinement, particles corresponding to each of these species were subjected to Bayesian polishing in RELION ${ }^{36}$ followed by homogeneous refinement in cryoSPARC coupled to CTF refinement. The monomeric S1-ACE2 complex was classified as in Extended Data Fig. 4a and refined using non-uniform refinement in cryoSPARC coupled to CTF refinement. The final particles from the S1-ACE2 complex were subjected to an unmasked refinement in RELION to better resolve less-ordered domains, with an overall lower global resolution (Extended Data Fig. 4b, c). Local resolution was estimated using blocres ${ }^{37}$ implemented in cryoSPARC. Maps were locally filtered and globally sharpened ${ }^{38}$ in cryoSPARC (Extended Data Figs. 5, 6).

\section{Model building}

The model for the monomeric S1-ACE2 complex was based on the previously determined crystal structure (PDB:6MOJ) ${ }^{24}$, with additional parts of the RBD and intermediate domain taken from a previous structure of the closed trimer (PDB: 6ZGE) ${ }^{26}$. Models of the trimer structures were built using structures from our previous study ${ }^{26}$ for the closed trimer (PDB: 6ZGE) and the one-erect-RBD structure (PDB: 6ZGG). The RBD-ACE2 parts of the model were built using the structure from the high resolution S1-ACE2 complex from this study. Models were manually adjusted using $\mathrm{COOT}^{39}$. The models of S1-ACE2 and the one-ACE2-bound closed structure were refined and validated using PHENIX real space refine ${ }^{40}$.
The other, lower resolution models were refined using NAMDINATOR ${ }^{41}$ and geometry minimization and validation in PHENIX (Extended Data Table 2). Measurements were made using Chimera ${ }^{42}, \mathrm{CCP} 4 \mathrm{MG}^{43}$ and $\mathrm{PISA}^{44}$, with structures aligned on the large helix of S2 (residues 986-1032).

\section{Reporting summary}

Further information on research design is available in the Nature Research Reporting Summary linked to this paper.

\section{Data availability}

Maps and models have been deposited in the Electron Microscopy Data Bank (EMD) and the Protein Data Bank (PDB) with the following accession codes: EMD-11681 and PDB 7A91 (dissociated S1 domain bound to ACE2 (non-uniform refinement)); EMD-11682 and PDB 7A92 (dissociated S1 domain bound to ACE2 (unmasked refinement)); EMD-11683 and PDB 7A93 (SARS-CoV-2 spike with two RBDs erect); EMD-11684 and PDB 7A94 (SARS-CoV-2 spike with one ACE2 bound); EMD-11685 and PDB 7A95 (SARS-CoV-2 spike with one ACE2 bound and one RBD erect in clockwise direction); EMD-11686 and PDB 7A96 (SARS-CoV-2 spike with one ACE2 bound and one RBD erect in anticlockwise direction); EMD-11687 and PDB 7A97 (SARS-CoV-2 spike with two ACE2 bound); EMD-11688 and PDB 7A98 (SARS-CoV-2 spike with three ACE2 bound).

31. Zheng, S. Q. et al. MotionCor2: anisotropic correction of beam-induced motion for improved cryo-electron microscopy. Nat. Methods 14, 331-332 (2017).

32. Scheres, S. H. W. RELION: implementation of a Bayesian approach to cryo-EM structure determination. J. Struct. Biol. 180, 519-530 (2012).

33. Rohou, A. \& Grigorieff, N. CTFFIND4: fast and accurate defocus estimation from electron micrographs. J. Struct. Biol. 192, 216-221 (2015).

34. Wagner, T. et al. SPHIRE-crYOLO is a fast and accurate fully automated particle picker for cryo-EM. Commun. Biol. 2, 218 (2019).

35. Punjani, A., Rubinstein, J. L., Fleet, D. J. \& Brubaker, M. A. cryoSPARC: algorithms for rapid unsupervised cryo-EM structure determination. Nat. Methods 14, 290-296 (2017).

36. Zivanov, J., Nakane, T. \& Scheres, S. H. W. A Bayesian approach to beam-induced motion correction in cryo-EM single-particle analysis. IUCrJ 6, 5-17 (2019).

37. Cardone, G., Heymann, J. B. \& Steven, A. C. One number does not fit all: mapping local variations in resolution in cryo-EM reconstructions. J. Struct. Biol. 184, 226-236 (2013).

38. Rosenthal, P. B. \& Henderson, R. Optimal determination of particle orientation, absolute hand, and contrast loss in single-particle electron cryomicroscopy. J. Mol. Biol. 333, 721-745 (2003).

39. Emsley, P., Lohkamp, B., Scott, W. G. \& Cowtan, K. Features and development of Coot. Acta Crystallogr. D 66, 486-501 (2010).

40. Adams, P. D. et al. PHENIX: a comprehensive Python-based system for macromolecular structure solution. Acta Crystallogr. D 66, 213-221 (2010).

41. Kidmose, R. T. et al. Namdinator - automatic molecular dynamics flexible fitting of structural models into cryo-EM and crystallography experimental maps. IUCrJ 6, 526-531 (2019).

42. Pettersen, E. F. et al. UCSF Chimera-a visualization system for exploratory research and analysis. J. Comput. Chem. 25, 1605-1612 (2004).

43. McNicholas, S., Potterton, E., Wilson, K. S. \& Noble, M. E. M. Presenting your structures: the CCP4mg molecular-graphics software. Acta Crystallogr. D 67, 386-394 (2011).

44. Krissinel, E. \& Henrick, K. Inference of macromolecular assemblies from crystalline state. J. Mol. Biol. 372, 774-797 (2007).

Acknowledgements We thank A. Nans of the Structural Biology Science Technology Platform for assistance with data collection, P. Walker and A. Purkiss of the Structural Biology Science Technology Platform and the Scientific Computing Science Technology Platform for computational support, and L. Calder, P. Cherepanov, G. Kassiotis and S. Kjaer for discussions. This work was funded by the Francis Crick Institute, which receives its core funding from Cancer Research UK (FCO01078 and FCO01143), the UK Medical Research Council (FC001078 and FC001143) and the Wellcome Trust (FC001078 and FC001143). P.X. is also supported by the 100 Top Talents Program of Sun Yat-sen University, the Sanming Project of Medicine in Shenzhen (SZSM201911003) and the Shenzhen Science and Technology Innovation Committee (grant no. JCYJ20190809151611269).

Author contributions D.J.B., A.G.W., P.X., C.R. and S.R.M. performed research, collected and analysed data; D.J.B., A.G.W., P.B.R., J.J.S. and S.J.G. conceived and designed research and wrote the paper.

Competing interests The authors declare no competing interests.

\section{Additional information}

Supplementary information is available for this paper at https://doi.org/10.1038/s41586-020 2772-0.

Correspondence and requests for materials should be addressed to D.J.B., A.G.W. or S.J.G. Peer review information Nature thanks Stephen Harrison and the other, anonymous, reviewer(s) for their contribution to the peer review of this work.

Reprints and permissions information is available at http://www.nature.com/reprints. 


\section{Article}
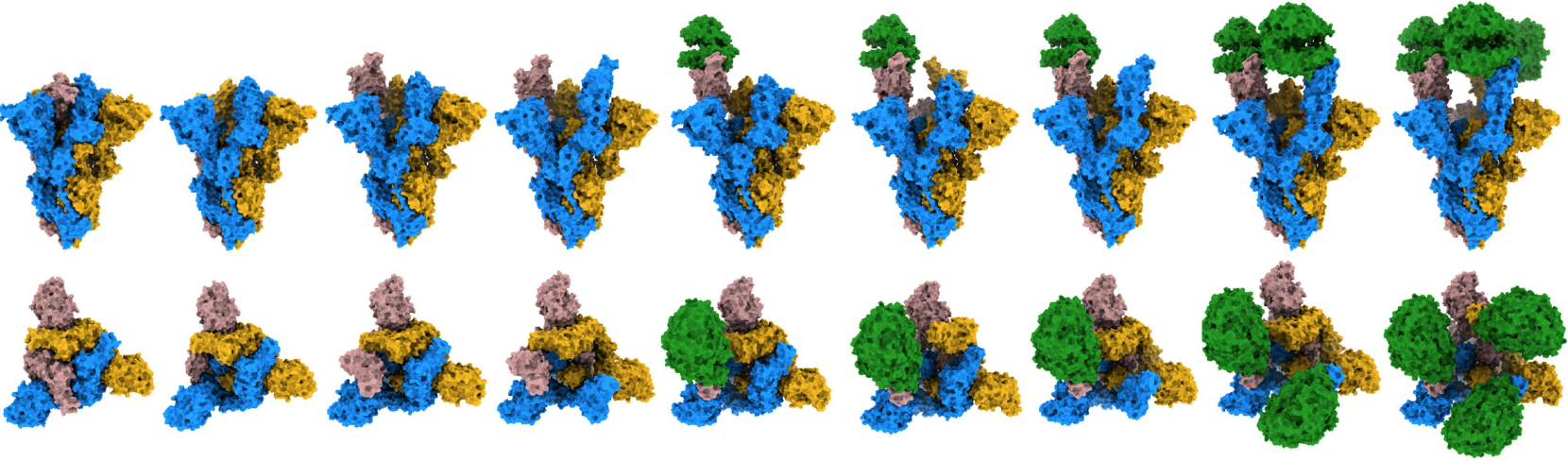

Closed

Intermediate

Open

Open

1 Bound

1 Bound RBD

1 Bound RBD

2 Bound

3 Bound

1 Erect RBD

1 Erect RBD

Clockwise

Anticlockwise

$\%$ of Total

Trimeric $\quad 11 \%$

$4 \%$

$16 \%$

$4 \%$

$28 \%$

$14 \%$

$7 \%$

$14 \%$

$3 \%$

Extended Data Fig. 1 |Surface representation of obtained structures. The three monomers of S in each trimer are coloured in blue, rosy brown and gold with ACE2 shown in green. Relative percentages of all trimeric S particles used to calculate electron microscopy maps are shown. 

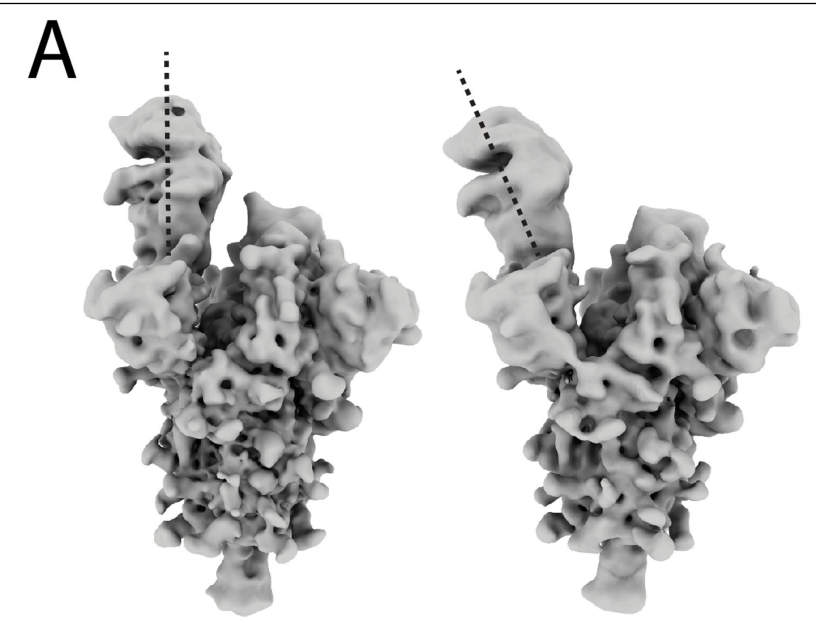

B
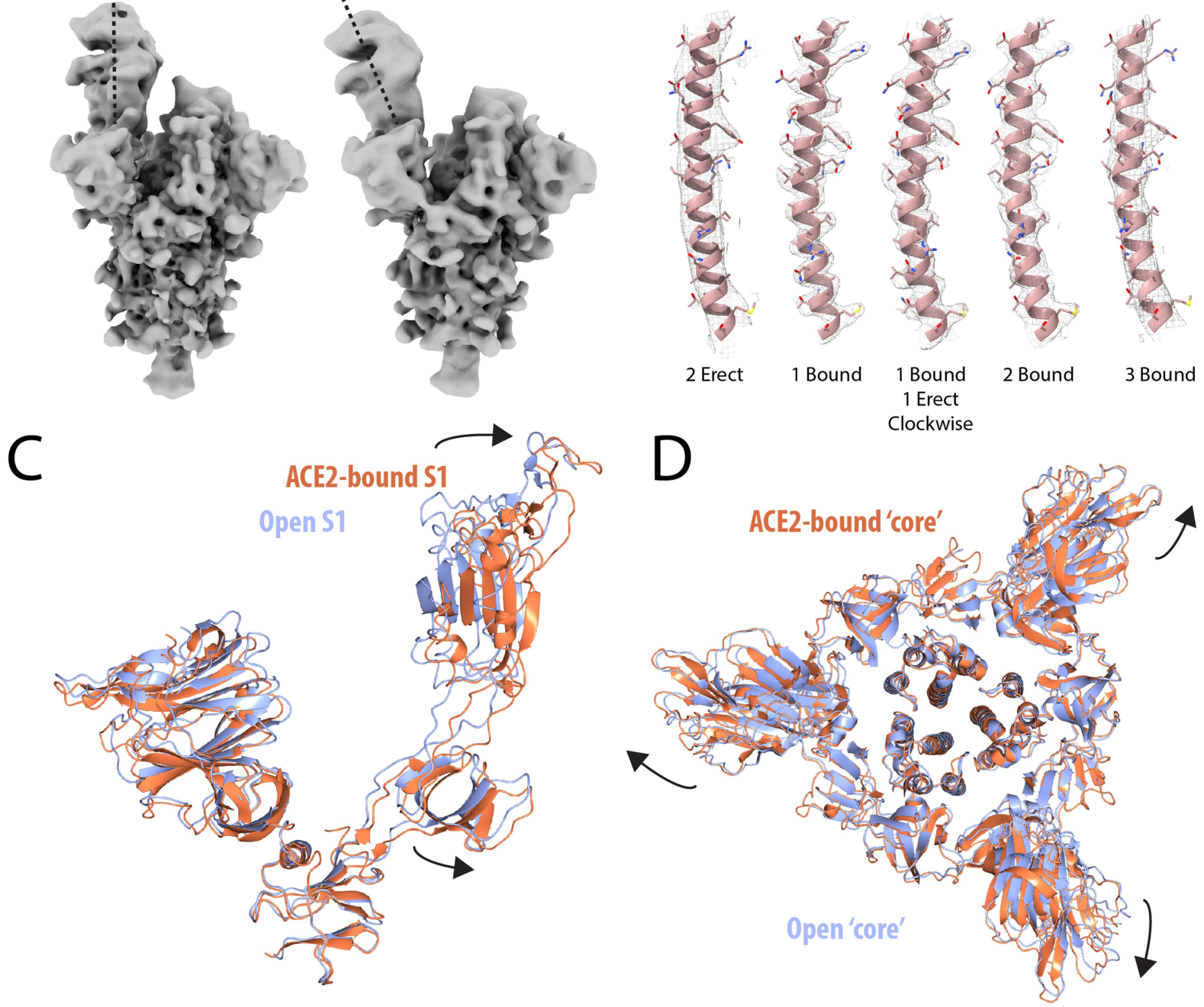

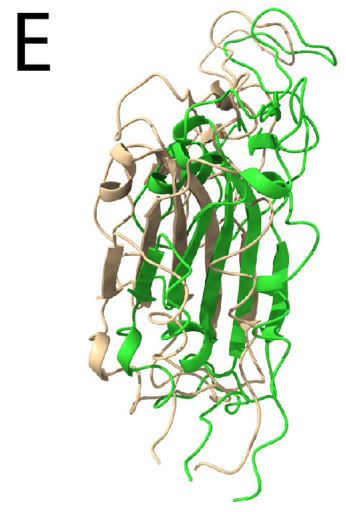

Unbound
1 Bound
Closed

\section{Bound RBD 1 \\ 2 Bound RBD 2}

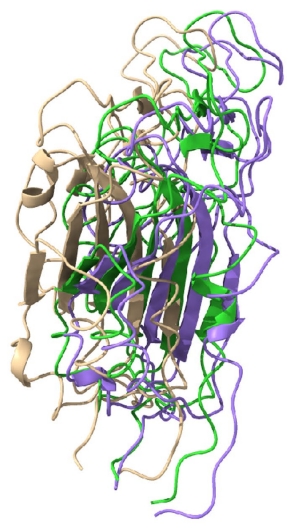

Unbound

Extended Data Fig. 2 | Features of the obtained spike structures. a, Two three-dimensional classes, obtained by further classification of the one-ACE2-bound closed state from Fig. 1, representative of the range of motion of the RBD with bound ACE2, tilting away from the trimer axis of the spike trimer. The tilt of the RBD and ACE2 is indicated with a dashed line. b, Representative density of different obtained electron microscopy maps for residues $996-1030$ of S2. Built model shown in pink, with EM density shown as a mesh.c, d, Comparison of spike structures for the open one-erect-RBD

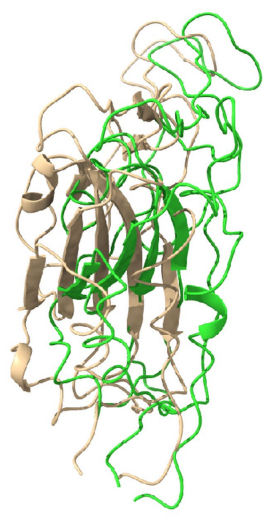

Unbound

\section{Bound \\ C105 Fab Bound RBD 1 C105 Fab Bound RBD 2}

structure (purple) with the one-ACE2-bound structure (orange).c, S1 domains shown to highlight domain shifts of the RBD and RBD-associated intermediate domain. d, Outwards movements of spike domains (excluding RBDs). e, Comparison of RBD displacements of one-bound, two-bound and three-bound RBDs after binding of ACE2 to the unbound open structure of the spike protein (beige). These are compared to the RBD displacement after binding of the $\mathrm{C} 105 \mathrm{Fab}$ fragment ${ }^{27}$, which binds at the ACE2 interface of the RBD (PDB: 6XCM). 

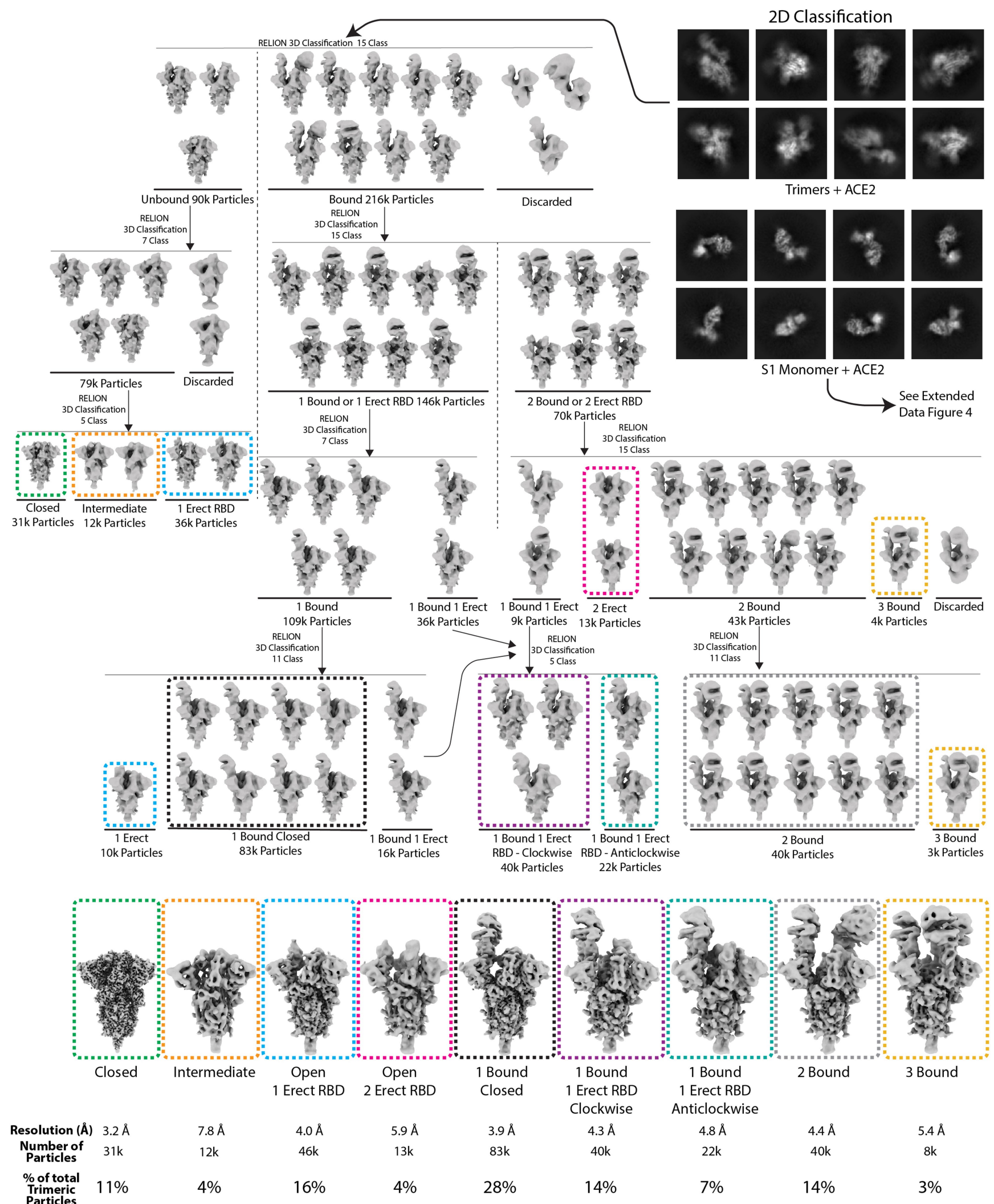

Extended Data Fig. 3 | Cryo-electron microscopy data processing scheme. Classes of particles used to obtain the final spike trimer structures, unbound and in complex with ACE2, are surrounded by a box of the same colour as the final maps shown at the bottom. The global resolution, final particle number and percentage for each trimer species are shown at the bottom. 
A

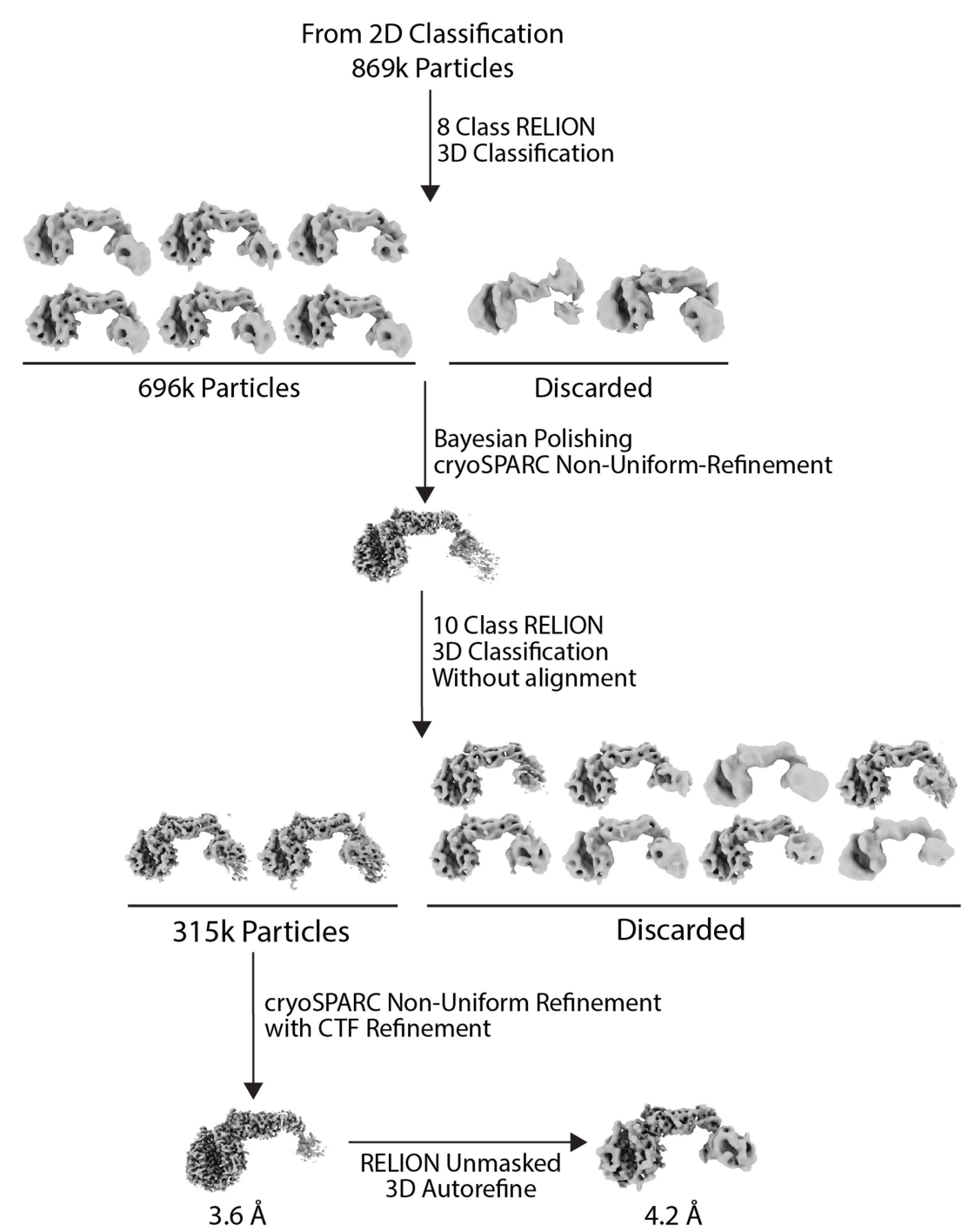

B
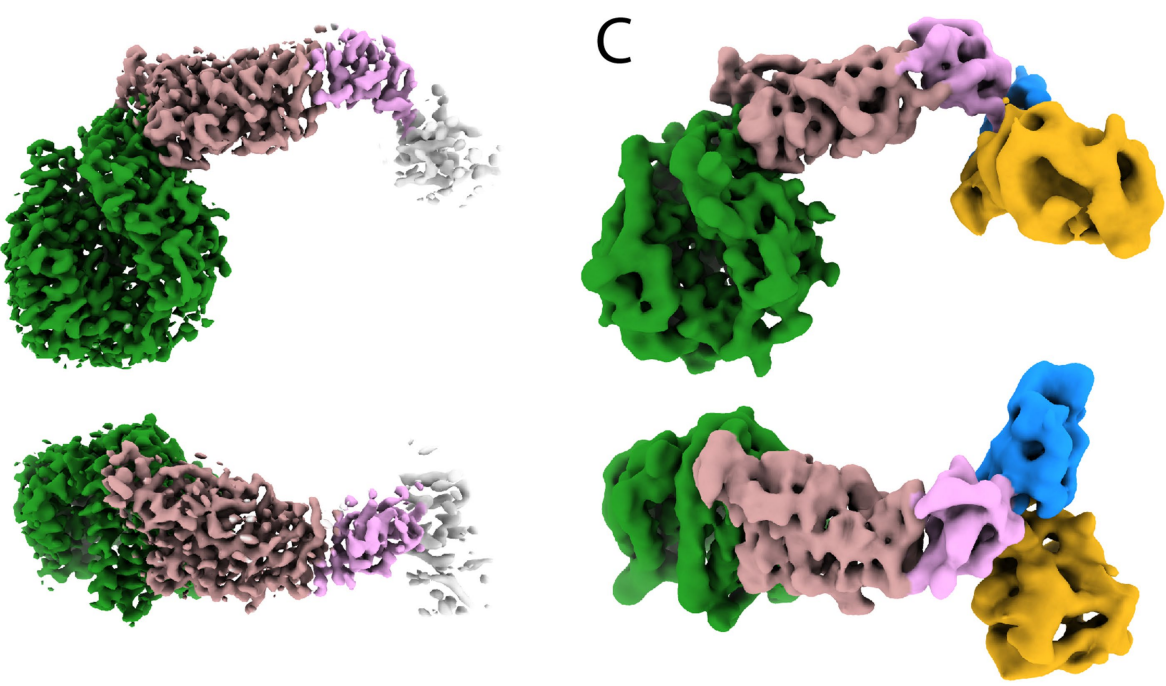

Extended Data Fig. 4 | Monomeric S1 bound to ACE2. a, Classification scheme for the S1-ACE2 complex.b, c, Maps are shown of orthogonal views of the non-uniform refinement (b) and unmasked refinement (c) of the final

particles. Domains are coloured as follows: green, ACE2; yellow, NTD; rosy brown, RBD; pink, RBD ganymede; blue, NTD ganymede; cream, disseminated S1 density in $\mathbf{b}$. 

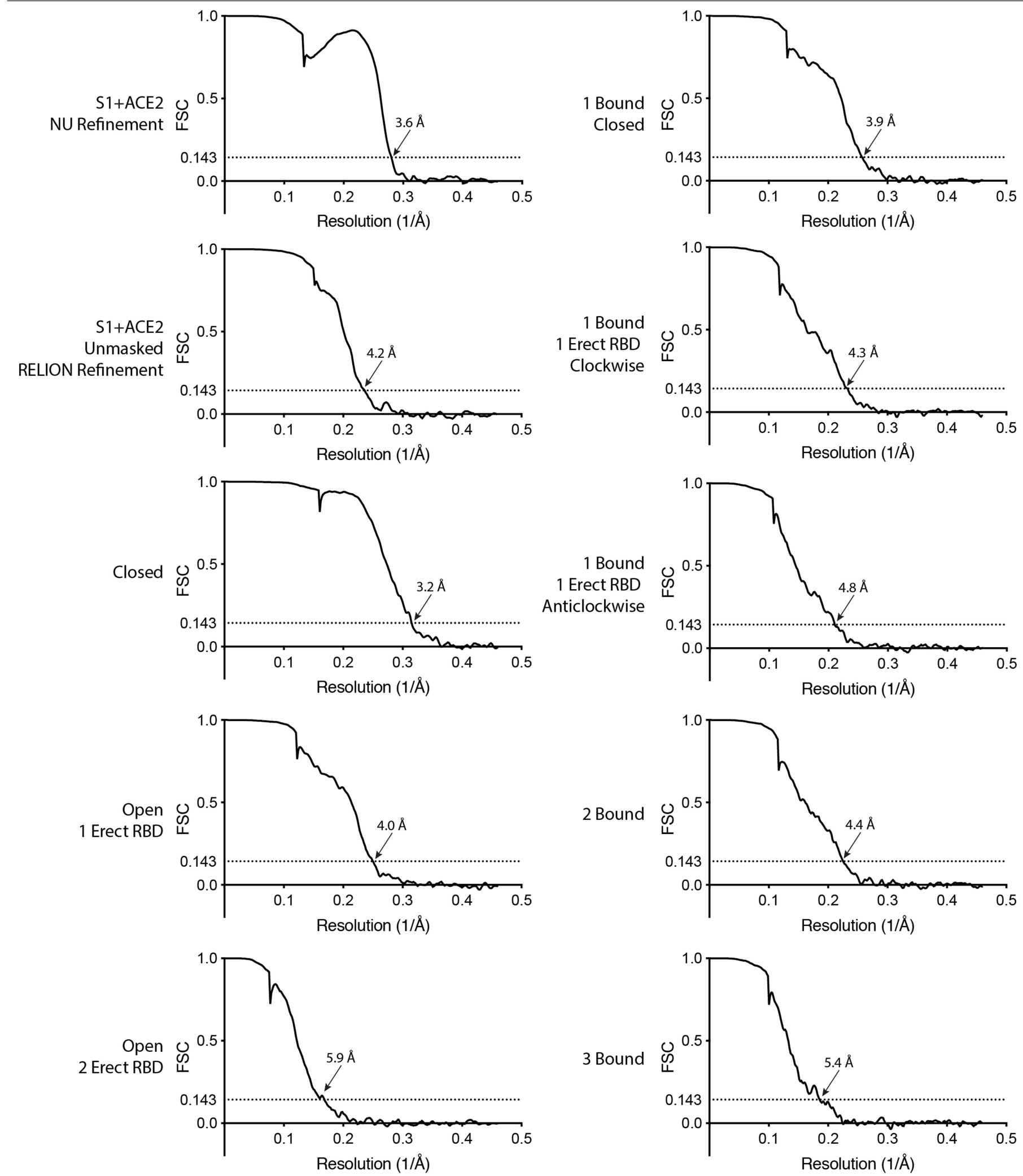

Extended Data Fig. 5 | Fourier shell correlation graphs for each of the determined structures. FSC, Fourier shell correlation. 

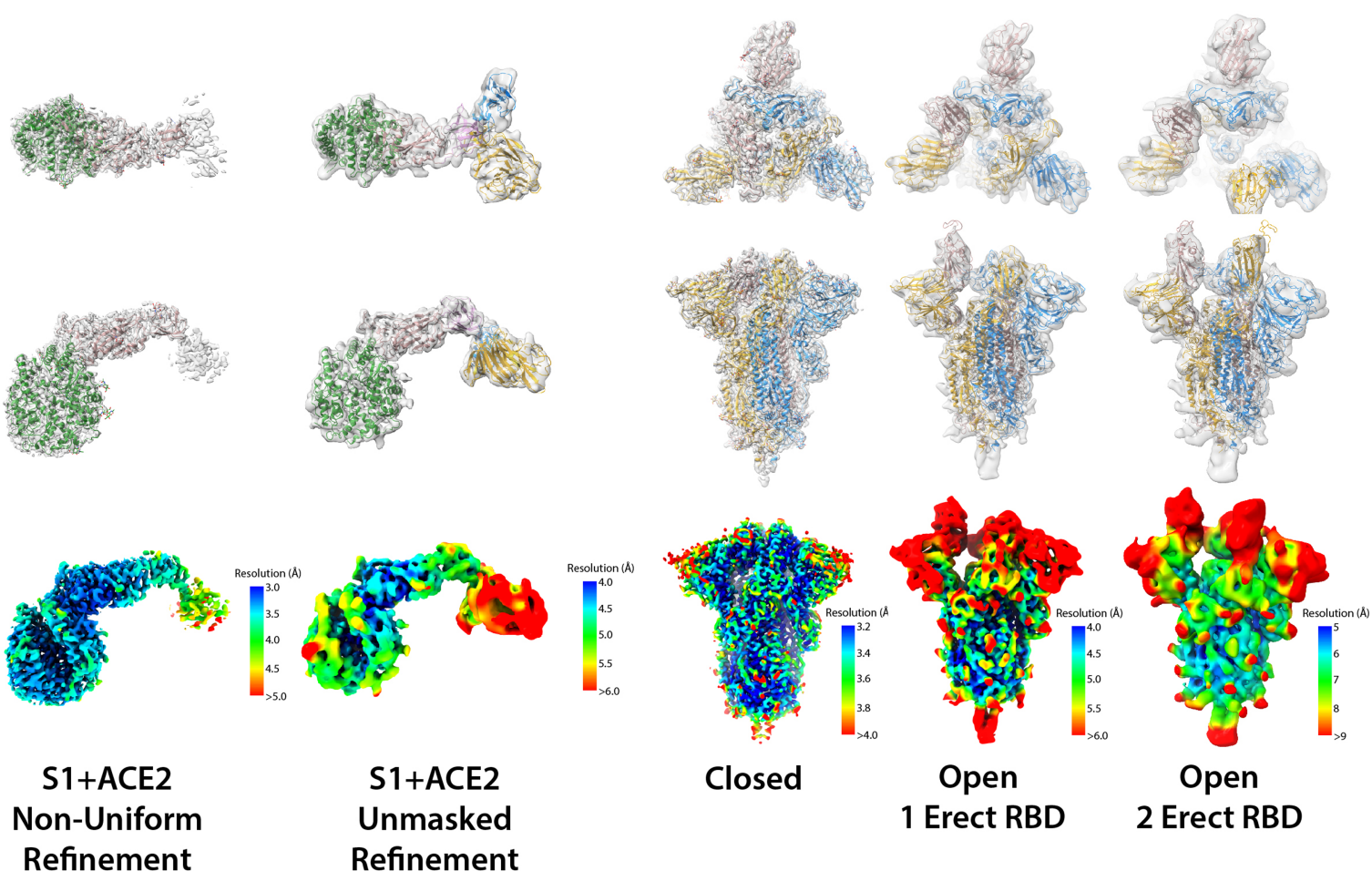

Closed
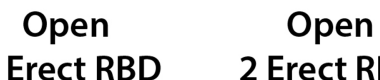

1 Erect RBD

\section{Erect RBD}
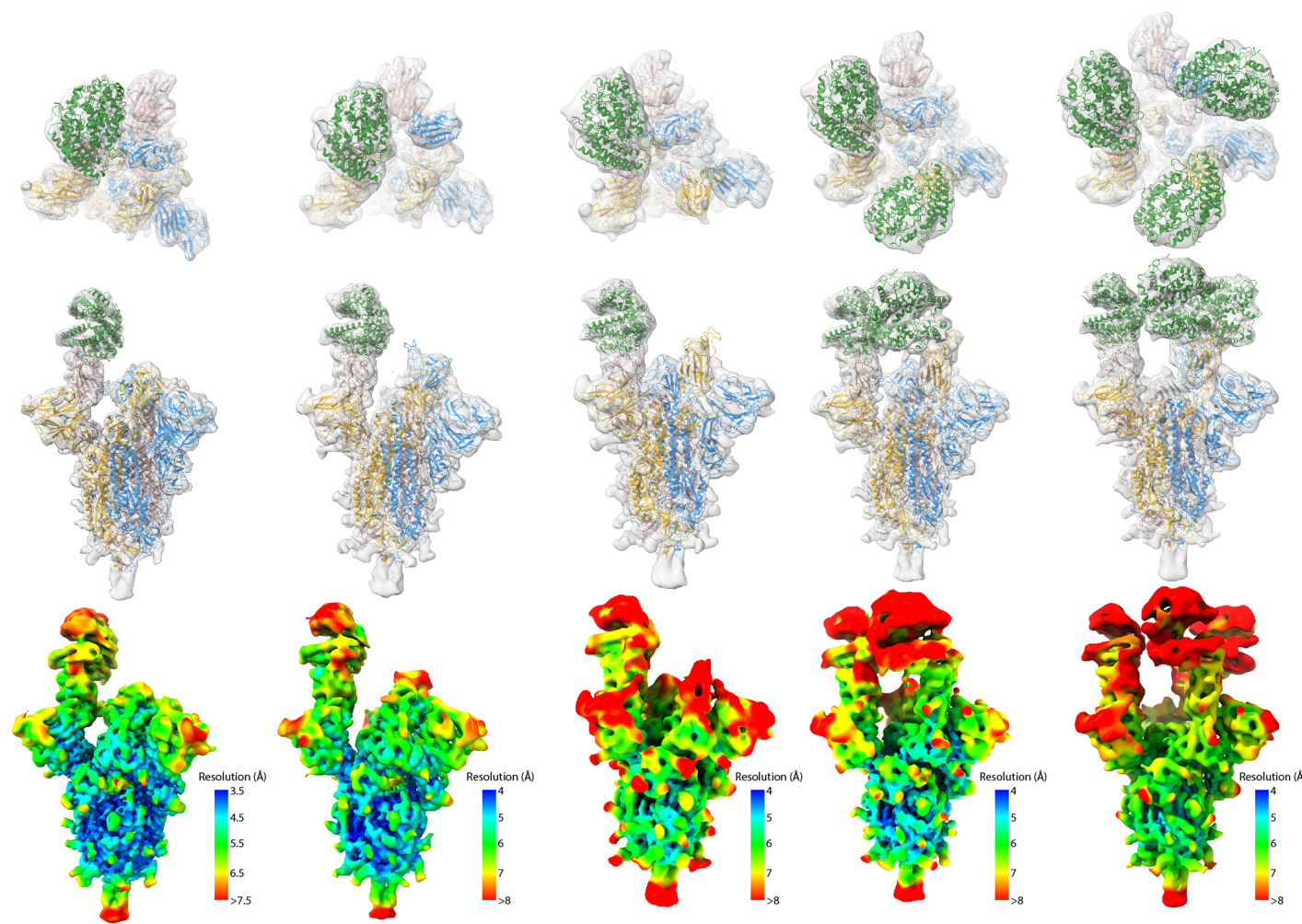

1 Bound
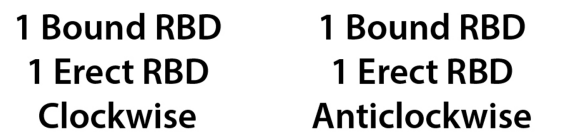

2 Bound

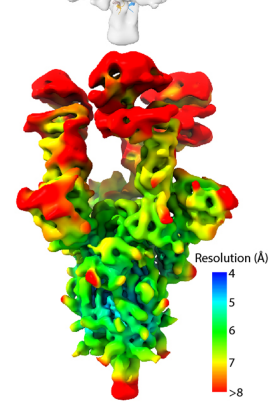

3 Bound

Extended Data Fig. 6 | Maps and models of determined structures. Top, orthogonal views of electron microscopy density (grey) and ribbon diagram representation of the models. Bottom, electron microscopy maps coloured by local resolution shown below. 


\section{Article}

Extended Data Table 1 | Buried interface surface area between monomers in different conformations

\begin{tabular}{lccc}
\hline & $\begin{array}{c}\text { Interface } \\
\text { A to B }\left(\AA^{2}\right)\end{array}$ & $\begin{array}{c}\text { Interface } \\
\text { A to C }\left(\AA^{2}\right)\end{array}$ & $\begin{array}{c}\text { Interface } \\
\mathrm{B} \text { to C }\left(\AA^{2}\right)\end{array}$ \\
\hline UNBOUND & & & \\
Closed & 5900 & 5900 & 5900 \\
Open & 4000 & 4500 & 4700 \\
RECEPTOR BOUND & & & \\
1ACE2-bound, closed & 3800 & 4200 & 4100 \\
1ACE2-bound, open & 3900 & 3900 & 4200 \\
2ACE2-bound & 3900 & 3900 & 4200 \\
3ACE2-bound & 3900 & 3900 & 3900 \\
\hline
\end{tabular}

Different confirmations of unbound and ACE2-bound trimers were analysed. The interface area was calculated using PISA. In the open and ACE2-bound conformations, chain A is the one to open first and to bind the receptor first, then $B$ follows, if the second RBD changes the conformation. Chain $B$ is the chain anticlockwise to $A$ when looking down the symmetry axis with the membrane-proximal part at the bottom. The unbound and three-ACE2-bound molecules are of $C_{3}$ symmetry. 
Extended Data Table 2 | Cryo-electron microscopy data collection, refinement and validation statistics

\begin{tabular}{|c|c|c|c|c|c|c|c|c|}
\hline & $\begin{array}{c}\text { S1+ACE2 Non- } \\
\text { uniform } \\
\text { Refinement } \\
\text { (EMDB-11681) } \\
\text { (PDB 7A91) }\end{array}$ & $\begin{array}{c}\text { S1+ACE2 } \\
\text { Unmasked } \\
\text { Refinement } \\
\text { (EMDB-11682) } \\
\text { (PDB 7A92) }\end{array}$ & $\begin{array}{c}2 \text { Erect RBD } \\
\text { (EMDB-11683) } \\
\text { (PDB 7A93) }\end{array}$ & $\begin{array}{l}1 \text { ACE2 Bound } \\
\text { Closed } \\
\text { (EMDB-11684) } \\
\text { (PDB 7A94) }\end{array}$ & $\begin{array}{l}1 \text { ACE2 Bound } \\
1 \text { Erect RBD } \\
\text { Clockwise } \\
\text { (EMDB-11685) } \\
\text { (PDB 7A95) }\end{array}$ & $\begin{array}{l}\text { 1 ACE2 Bound } \\
1 \text { Erect RBD } \\
\text { Anticlockwise } \\
\text { (EMDB-11686) } \\
\text { (PDB 7A96) }\end{array}$ & $\begin{array}{c}2 \text { ACE2 Bound } \\
\text { (EMDB-11687) } \\
\text { (PDB 7A97) }\end{array}$ & $\begin{array}{c}\text { 3ACE2 Bound } \\
\text { (EMDB-11688) } \\
\text { (PDB 7A98) }\end{array}$ \\
\hline \multicolumn{9}{|l|}{$\begin{array}{l}\text { Data collection and } \\
\text { processing }\end{array}$} \\
\hline Voltage $(\mathrm{kV})$ & 300 & 300 & 300 & 300 & 300 & 300 & 300 & 300 \\
\hline Electron exposure $\left(\mathrm{e}-/ \AA^{2}\right)$ & 54.4 & 54.4 & 54.4 & 54.4 & 54.4 & 54.4 & 54.4 & 54.4 \\
\hline Defocus range $(\mu \mathrm{m})$ & -1.5 to -3.0 & -1.5 to -3.0 & -1.5 to -3.0 & -1.5 to -3.0 & -1.5 to -3.0 & -1.5 to -3.0 & -1.5 to -3.0 & -1.5 to -3.0 \\
\hline Pixel size $(\AA)$ & 1.08 & 1.08 & 1.08 & 1.08 & 1.08 & 1.08 & 1.08 & 1.08 \\
\hline Symmetry imposed & $\mathrm{C} 1$ & $\mathrm{C} 1$ & $\mathrm{C} 1$ & $\mathrm{C} 1$ & $\mathrm{C} 1$ & $\mathrm{C} 1$ & $\mathrm{C} 1$ & $\mathrm{C} 3$ \\
\hline Final particle images (no.) & $315 \mathrm{k}$ & $315 \mathrm{k}$ & $13 \mathrm{k}$ & $83 \mathrm{k}$ & $40 \mathrm{k}$ & $22 \mathrm{k}$ & $40 \mathrm{k}$ & $8 \mathrm{k}$ \\
\hline Map resolution $(\AA)$ & 3.6 & 4.2 & 5.9 & 3.9 & 4.3 & 4.8 & 4.4 & 5.4 \\
\hline $\begin{array}{c}\text { FSC threshold }=0.143 \\
\text { Map resolution range }(\AA)\end{array}$ & $3-5$ & $4-6$ & $5-9$ & $3.5-7.5$ & $4-8$ & $4-8$ & $4-8$ & $5-8$ \\
\hline \multicolumn{9}{|l|}{ Refinement } \\
\hline Initial model used (PDB code) & $6 \mathrm{MOJ}$ & - & - & - & - & - & - & - \\
\hline $\begin{array}{l}\text { Model resolution }(\AA) \\
\text { FSC threshold }=0.5\end{array}$ & 3.6 & 4.4 & 6.7 & 4.0 & 4.4 & 4.9 & 4.6 & 6.1 \\
\hline $\begin{array}{l}\text { Map sharpening } B \text { factor }\left(\AA^{2}\right) \\
\text { Model composition }\end{array}$ & -98.4 & -158.9 & -169.6 & -75.0 & -64.7 & -66.1 & -67.2 & -119.6 \\
\hline Non-hydrogen atoms & 6915 & 10040 & 25243 & 30475 & 29904 & 29828 & 34645 & 39456 \\
\hline Protein residues & 839 & 1231 & 3221 & 3825 & 3794 & 3783 & 4373 & 4962 \\
\hline Ligands & 10 & 10 & - & 24 & - & - & - & - \\
\hline \multicolumn{9}{|l|}{ R.m.s. deviations } \\
\hline Bond lengths $(\AA)$ & 0.006 & 0.007 & 0.004 & 0.007 & 0.004 & 0.004 & 0.004 & 0.004 \\
\hline \multicolumn{8}{|l|}{ Validation } & 0.918 \\
\hline MolProbity score & 1.46 & 1.55 & 1.62 & 1.57 & 1.71 & 1.75 & 1.81 & 1.85 \\
\hline Clashscore & 3.25 & 3.71 & 2.83 & 3.38 & 3.48 & 3.88 & 4.85 & 5.71 \\
\hline Poor rotamers $(\%)$ & 0.81 & 1.10 & 0.85 & 0.90 & 0.90 & 0.82 & 0.23 & 0.83 \\
\hline \multicolumn{9}{|l|}{ Ramachandran plot } \\
\hline Favored $(\%)$ & 95.07 & 94.81 & 90.11 & 93.16 & 88.97 & 89.21 & 89.70 & 90.23 \\
\hline Allowed (\%) & 4.93 & 5.02 & 9.89 & 6.84 & 11.03 & 10.77 & 10.28 & 9.65 \\
\hline Disallowed (\%) & 0.00 & 0.16 & 0.00 & 0.00 & 0.00 & 0.03 & 0.02 & 0.12 \\
\hline
\end{tabular}




\section{Reporting Summary}

Nature Research wishes to improve the reproducibility of the work that we publish. This form provides structure for consistency and transparency in reporting. For further information on Nature Research policies, see Authors \& Referees and the Editorial Policy Checklist.

\section{Statistics}

For all statistical analyses, confirm that the following items are present in the figure legend, table legend, main text, or Methods section.

n/a Confirmed

\ $\square$ The exact sample size $(n)$ for each experimental group/condition, given as a discrete number and unit of measurement

Х $\square$ A statement on whether measurements were taken from distinct samples or whether the same sample was measured repeatedly

$\square$ The statistical test(s) used AND whether they are one- or two-sided

$\triangle \square$ Only common tests should be described solely by name; describe more complex techniques in the Methods section.

Х $\square$ A description of all covariates tested

Х $\square$ A description of any assumptions or corrections, such as tests of normality and adjustment for multiple comparisons

$\searrow$ A full description of the statistical parameters including central tendency (e.g. means) or other basic estimates (e.g. regression coefficient)

AND variation (e.g. standard deviation) or associated estimates of uncertainty (e.g. confidence intervals)

$\searrow \square$ For null hypothesis testing, the test statistic (e.g. $F, t, r$ ) with confidence intervals, effect sizes, degrees of freedom and $P$ value noted

Х Give $P$ values as exact values whenever suitable.

Х $\square$ For Bayesian analysis, information on the choice of priors and Markov chain Monte Carlo settings

Х $\square$ For hierarchical and complex designs, identification of the appropriate level for tests and full reporting of outcomes

Х $\square$ Estimates of effect sizes (e.g. Cohen's $d$, Pearson's $r$ ), indicating how they were calculated

Our web collection on statistics for biologists contains articles on many of the points above.

\section{Software and code}

Policy information about availability of computer code

Data collection CryoEM data collected using Thermo Scientific EPU v2.7

Data analysis CryoEM data processed using following packages: RELION-3.1, cryoSPARC v2.14, CTFfind4 v.4.1.10, MotionCor2 v.1.2.6, crYOLO v1.4,

Coot v.0.9, PHENIX v.1.17, UCSF Chimera v.1.12, UCSF ChimeraX v.0.5, CCP4MG v2.10, PISA v1.52

For manuscripts utilizing custom algorithms or software that are central to the research but not yet described in published literature, software must be made available to editors/reviewers. We strongly encourage code deposition in a community repository (e.g. GitHub). See the Nature Research guidelines for submitting code \& software for further information.

\section{Data}

Policy information about availability of data

All manuscripts must include a data availability statement. This statement should provide the following information, where applicable:

- Accession codes, unique identifiers, or web links for publicly available datasets

- A list of figures that have associated raw data

- A description of any restrictions on data availability

Maps and models have been deposited in the Electron Microscopy Data Bank, http://www.ebi.ac.uk/pdbe/emdb/ and the Protein Data Bank, https:// www.ebi.ac.uk/pdbe/ with the following accession codes: EMD-11681 and PDB 7 A91 (Dissociated S1 domain bound to ACE2 [Non-Uniform Refinement]); EMD-11682 and PDB 7A92 (Dissociated S1 domain bound to ACE2 [Unmasked Refinement]); EMD-11683 and PDB 7A93 (SARS-CoV-2 S with 2 RBDs Erect); EMD-11684 and PDB 7A94 (SARS-CoV-2 S with 1 ACE2 Bound); EMD-11685 and PDB 7 A95 (SARS-CoV-2 S with 1 ACE2 Bound and 1 RBD Erect in Clockwise Direction); EMD-11686 and PDB 7A96 (SARS-CoV-2 S with 1 ACE2 Bound and 1 RBD Erect in Anticlockwise Direction); EMD-11687 and PDB 7A97 (SARS-CoV-2 S with 2 ACE2 Bound); EMD-11688 and PDB 7 A98 (SARS-CoV-2 S with 3 ACE2 Bound). 


\section{Field-specific reporting}

Please select the one below that is the best fit for your research. If you are not sure, read the appropriate sections before making your selection. \ Life sciences

$\square$ Behavioural \& social sciences Ecological, evolutionary \& environmental sciences

For a reference copy of the document with all sections, see nature.com/documents/nr-reporting-summary-flat.pdf

\section{Life sciences study design}

All studies must disclose on these points even when the disclosure is negative. Sample size $\quad \begin{aligned} & \text { All cryoEM datasets consist of several thousand images. The number of images were sufficient to achieve the reported resolution, according } \\ & \text { to the most commonly reported resolution measure in cryoEM described in Rosenthal and Henderson 2003, as cited in the manuscript }\end{aligned}$

Data exclusions CryoEM single particles were included and excluded within the image processing workflow using standard image processing techniques such as 2D and 3D classifications, as detailed in Extended Data Figures 3 and 4.

Replication Structures were determined using independent half datasets, according to standard procedures in cryoEM. Images were collected from three independent replicate prepared grids, which all produced similar images both by low resolution visual inspection and high resolution class averages. There were no unsuccessful replications.

Randomization Not applicable to this study, as samples were not assigned to experimental groups and data were collected and processed according to standard techniques for cryoEM.

Blinding

Not applicable to this study, as there was no experimental group allocation in data collection and analysis.

\section{Reporting for specific materials, systems and methods}

We require information from authors about some types of materials, experimental systems and methods used in many studies. Here, indicate whether each material, system or method listed is relevant to your study. If you are not sure if a list item applies to your research, read the appropriate section before selecting a response.

Materials \& experimental systems

\begin{tabular}{l|l}
\hline Ia & Involved in the study \\
$\square$ & Antibodies \\
$\square$ & $\square$ Eukaryotic cell lines \\
$\square$ & $\square$ Clinical data
\end{tabular}

Methods

n/a Involved in the study

$\bigotimes \square$ ChIP-seq

$\triangle \square$ Flow cytometry

\ $\square$ MRI-based neuroimaging

\section{Eukaryotic cell lines}

\section{Policy information about cell lines}

Cell line source(s)

expi293F cells were purchased from Thermo Scientific and used for protein expression

Authentication

Cell line used was not authenticated

Mycoplasma contamination

Cell line was not tested for mycoplasma contamination

Commonly misidentified lines

(See ICLAC register) 